\title{
A continuous high voltage electrostatic field system for thawing food materials
}

\author{
Ehsan Movahedi, ${ }^{1}$ Morteza Sadeghi, ${ }^{1}$ Amin Allah Masoumi, ${ }^{1}$ Naser Hamdami \\ ${ }^{1}$ Department of Biosystems Engineering; ${ }^{2}$ Department of Food Science and Technology, College of Agriculture, \\ Isfahan University of Technology, Isfahan, Iran
}

\begin{abstract}
A laboratory continuous high voltage electrostatic field thawing system was designed and constructed in accordance with standards for food machinery. A corona discharge in wire-plate electrodes was considered for the system and a stainless-steel sheet was utilized as conveyor belt. A corona flow was created by using high voltage of 15,20 , and $25 \mathrm{kV}$ and electrode gaps of 6,8 , and $10 \mathrm{~cm}$. To evaluate the system, frozen tylose MH4000 gel with a moisture content of $77 \%$ w.b. as a meat analog was thawed from -10 to $0^{\circ} \mathrm{C}$. The thawing duration, which was mostly aimed at increasing the temperature of a control sample (no electrostatic field), considerably decreased when the gel was thawed in the presence of high voltage. High voltage and electrode gap showed a significant effect on both thawing duration and specific energy consumption $(\mathrm{P}<0.0001)$. The thawing duration decreased significantly by decreasing the electrode gap and increasing the applied voltage. In terms of energy consumption, the best condition (minimum specific energy consumption of $10.33 \mathrm{~kJ} \mathrm{~kg}^{-1}$ ) was obtained for $15 \mathrm{kV}$, and an electrode gap of $10 \mathrm{~cm}$.
\end{abstract}

Correspondence: Morteza Sadeghi, Department of Biosystems Engineering, College of Agriculture, Isfahan University of Technology, Isfahan 84156-83111, Iran.

Tel.: +98.3133913508 - Fax: +98.3133912254.

E-mail: sadeghimor@iut.ac.ir

Key words: Continuous thawing; corona flow; electrostatics; Tylose gel; wire-plate arrangement.

Acknowledgements: this work was supported by Isfahan University of Technology which is acknowledged.

Conflict of interests: the authors declare no potential conflict of interests.

Received for publication: 17 February 2021.

Accepted for publication: 22 April 2021.

(C) Copyright: the Author(s), 2021

Licensee PAGEPress, Italy

Journal of Agricultural Engineering 2021; LII:1163

doi:10.4081/jae.2021.1163

This article is distributed under the terms of the Creative Commons Attribution Noncommercial License (by-nc 4.0) which permits any noncommercial use, distribution, and reproduction in any medium, provided the original author(s) and source are credited.

\section{Introduction}

Freezing is one of the most important processes for preserving vegetables, meats, and some fruits. Most frozen foods should be first thawed. Conventional thawing methods consist in exposing the frozen material to air, dipping it in cold water, tap water, hot water, as well as keeping it in the refrigerator (Eastridge and Bowker, 2011). However, these methods are time-consuming and may deteriorate food quality, especially in the case of meat thawing. There are also some limitations in other methods, such as microwave thawing. This method usually does not ensure uniform thawing and some parts of the thawed food may be cooked (Taher and Farid, 2001).

In most thawing methods, the process is performed in a batch mode in which the food is placed in a container and is thawed. While for thawing a large amount of material, the continuous movement of the product could be more suitable regarding its health and better efficiency over the batch mode. Hence, many studies were conducted on the development of new efficient methods to improve the freezing and thawing processes (Ould Ahmedou and Havet, 2009).

Using the conventional methods like passing an airflow at low speed over the frozen food, when in large pieces, like in the case of meat or fish, is time consuming. In this method, thawing is accomplished by convective heat transfer to the product on its surface, but deeper frozen layers have a low thermal conductivity. To overcome this issue, the airflow rate can be increased to enhance the heat transfer rate, but the energy consumption increases significantly. Hence, in recent studies new thawing methods based on ultrasound, ohmic, high pressure, pulsed electric fields, magnetic nanoparticles plus heating, radio frequency and high voltage electrostatic fields (HVEF) are used. Except for HVEF thawing, all other methods are time consuming and energy intensive (Cai et al., 2019). Therefore, there is a need to develop methods that can decrease energy consumption and thawing duration without damaging the food and causing microbial growth, as well as other disadvantages associated with physical and chemical changes in the products (Yar et al., 2015).

HVEF thawing was applied in a few studies at a laboratory scale. In a study the energy required for HVEF thawing was 0 to $190 \mathrm{~kJ} \mathrm{~kg}^{-1}$, which was less than the $1100 \mathrm{~kJ} \mathrm{~kg}^{-1}$ needed for microwave thawing (He et al., 2014). HVEF thawing caused fewer physicochemical and structural changes of myofibrillar proteins, total microbial counts, volatile basic nitrogen, and other meat parameters compared with traditional thawing methods. Jia et al. (2017) confirmed that HVEF thawing (voltage range: -25 to $0 \mathrm{kV}$ ) did not cause any protein oxidation and the dynamic rheological analysis, protein aggregation, and gel texture analyses showed better results compared with air thawing of frozen rabbit meat. In addition, there was no significant change in the structure of myofibrillar proteins. He et al. (2013) also reported that the 
HVEF treatment could reduce the total microbial count in thawed pork tenderloin meat by 0.5 to $1 \log \mathrm{CFU} / \mathrm{g}$ in a temperature range between -5 to $0{ }^{\circ} \mathrm{C}$. From the above, the HVEF is a suitable thawing technology for thawing meats, once parameters are optimized. Therefore, the advantages of HVEF thawing are low energy consumption, low generation of ozone that prevents an increase in the microbial load during the process, non-thermal thawing, and the use of simple equipment (Shivashankara et al., 2004). The systems used in the previous HVEF studies include a plate electrode and a needle or wire electrodes located on a specified air gap, and the frozen material is placed between two conductive electrodes on the plate. The electrodes are then connected to a high voltage source, and the voltage level is increased to a certain value for which no electrical discharge occurs (Xiangli et al., 2013). In such systems, when the voltage is applied to the needle or the wire electrodes, the air around them is ionized and the ions move toward the plate electrode connected to the other pole. During the movement of the ions towards the plate electrode, due to their collision with air molecules, the momentum of the ions is transferred to the air molecules. As a result of this collision, a mass of ionized air flows which is called corona wind or secondary flow is generated. The needle or the wire electrode is called corona electrode. The choice of the type of the corona electrodes mainly depends on the geometry of the chamber and the operation of the system. HVEF thawing systems with some needles and plate electrodes are operated by using AC or DC voltages. These systems are highly efficient for thawing the frozen material (Xie and Hua, 2001; Yaxiang et al., 2009). Thawing of some foods such as tuna, beef, and eggs was studied using HVEF at low ambient temperatures in the range of 3 to $3^{\circ} \mathrm{C}$ in a batch mode (Ohtsuki, 1991). The results showed that the thawing duration was reduced by one-quarter to one-third, compared to non-field conditions. Thawing of chicken thighs by HVEF showed that this method could significantly reduce the thawing duration at $-3^{\circ} \mathrm{C}$ and keep the product fresh compared to the conventional storage method in the refrigerator (Hsieh et al., 2010). Another study on frozen pork tenderloin revealed that the use of the HVEF with an electrode gap (EG) of $5 \mathrm{~cm}$ and applied voltages of 6,8 , and $10 \mathrm{kV}$ significantly increased the rate of thawing (Xiangli et al., 2013). Also, the investigation of thawing characteristic of frozen tofu under high-voltage alternating electric field revealed that as voltage increased, the thawing duration decreased (Deng et al., 2017). Ding et al. (2018) obtained similar results with frozen tofu under HVEF assisted thawing. They showed that the thawing duration of frozen tofu decreased with the increase of the voltage and it was highly related to the configuration and distance of the electrodes. Also, the electric parameters had a remarkable effect on thawing loss and thawing duration, when centre temperatures of frozen tofu decreased from -2 to $0^{\circ} \mathrm{C}$.

Yaxiang et al. (2011) studied the effects of different high voltages, EGs and distances between the adjacent needle electrodes on the thawing rate of ice and the energy consumption of the process in the batch mode. Based on the thawing rate curve of ice versus the distance between two needle electrodes, the thawing rate reached its maximum, when the distance between two adjacent electrodes was in the range of $6 \mathrm{~cm}$.

Despite the promising studies on the thawing of the food materials in a batch mode, to the best of the authors' knowledge, there is no study in the literature about HVEF thawing in the continuous mode. This study was aimed to design and construct a continuous system for feasibility investigation of HVEF thawing of meat at a laboratory scale. Some advantages of this type of HVEF thawing system could be thawing of frozen materials continuously as well as a reduction of time and cost for high volume thawing of food materials.

\section{Materials and methods}

In this study, a laboratory continuous HVEF thawing system was designed in CATIA software (Dassault system, CATIA V5R20). Afterwards, it was constructed as a type of tunnel according to food machinery standards. The thawing process is carried out by flowing the frozen material through this system under HVEF. Figure 1 shows the schematic of the system and its different parts.

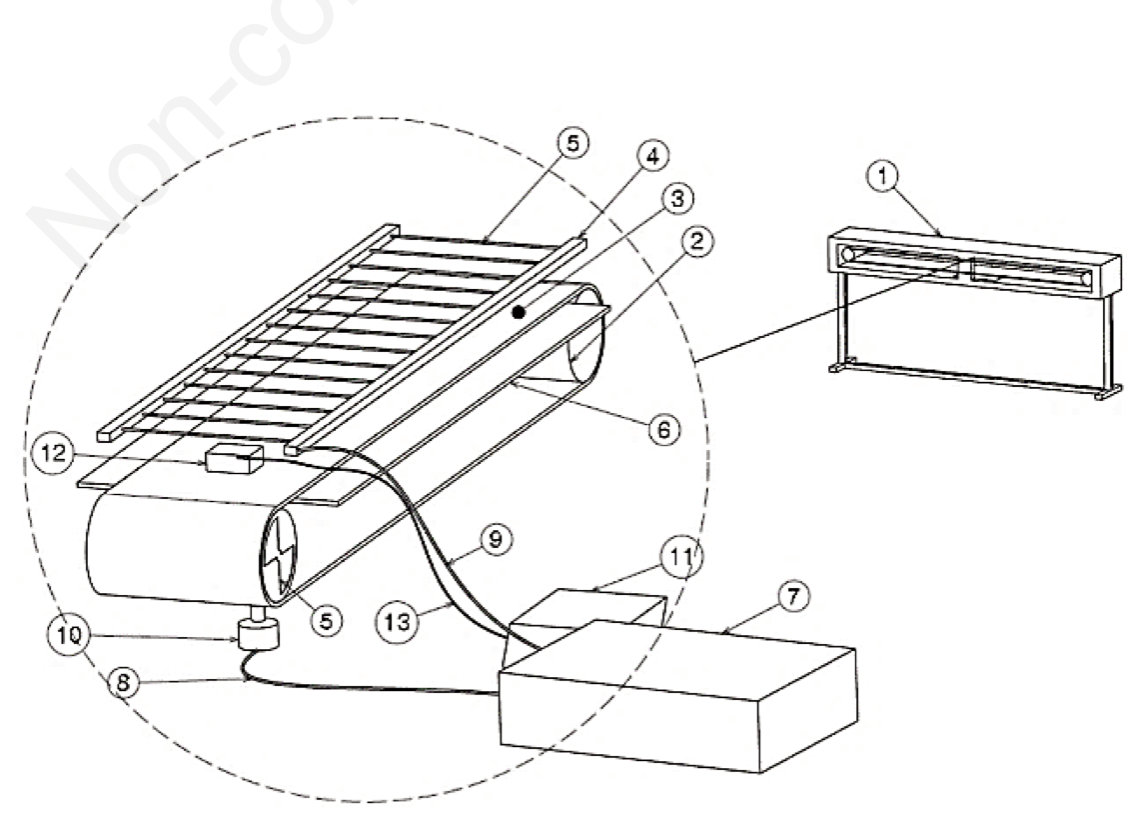

Figure 1. Schematic of the continuous high voltage electrostatic field thawing system: 1) complete device, 2) driver roller, 3) stainless steel conveyor belt, 4) aluminium rail, 5) wire electrode, 6) glass holder plate, 7) high-voltage power supply, 8 and 9) connections of power supply wires to the poles, 10) carbon electrode in contact with conveyor roller, and 11) temperature sensor. 
It should be noted that thawing under the HVEF is a complex process. The effect of the corona wind flow on such a system is affected by the geometry of the positive and negative electrodes (corona electrodes and ground electrode), the type of voltage (AC or DC), the voltage value, the distances between the two adjacent electrodes, and the EG and the presence of the initial wind flow in the system (Akishev et al., 2003; Lin and Adamiak, 2008). Since the applied voltage to the electrodes of the system is in kilovolts, it was necessary to insulate all electric conductive parts to ensure the safety of the user, when in contact with the high-voltage electrodes. Therefore, in order to have the optimal mechanism for the system, polyamide, glass, and compact plastic were used as insulation materials. The conductive components such as pole electrodes were also selected in accordance with the standards of food industry machines and were made of stainless steel 304 . The structure of the device and the geometry of its components along with their design details are described below.

\section{Corona electrodes}

Regarding the physics of the system, the frozen food moved inside a tunnel, while it was thawed. In order to facilitate the adjustment of electrodes distances, and their distribution over the channel and to ensure construction convenience, all the corona electrodes were considered as $0.5 \mathrm{~mm}$-diameter stainless steel wires (Lin and Adamiak, 2008). All the electrodes were mounted along the tunnel at specific intervals and attached transversally to the top of the tunnel in the form of a ladder (Figure 1). In order to change the EG, the height of the two rails could be adjusted from the ground electrode. The electric field created between the corona electrodes and the ground electrod was calculated by Eq. (1):

$$
E=\frac{V}{d}
$$

where, $V$ was the voltage, and $d$ was the electrode gap.

In the system, the values of the applied voltage and the electrode gap are adjustable in the range of 0 to $70 \mathrm{kV}$ and 1 to $20 \mathrm{~cm}$, respectively. For the experiments, the applied voltage was considered in the range of 15 to $25 \mathrm{kV}$ and the EG was in the range of 6 to $10 \mathrm{~cm}$ (Yaxiang et al., 2011; Deng et al., 2017). Since the electric field created between the two poles was inversely proportional to the distance between them according to Eq. (1), it was necessary for the wire electrodes to be transverse, smooth and straight and with no deflection. Also, the electrode should be able to move on the rail so as to adjust the distance between the two adjacent wires. Eventually, one of the rails of the formed ladder was connected to the positive pole of the high voltage power supply. The ionization region around each wire after applying the voltage, known as corona crescent radius, was calculated using Eq. (2) (Guo et al., 2014):

$r_{i}=r+0.03 \sqrt{ } r$

where $r$ is the wire radius and $r_{i}$ is the ionization radius.

The ionization radius increased, as the applied voltage increased. Given the small ionization radius, this area can be ignored. Therefore, the distance between two adjacent electrodes in the system was approximately selected to be 100 times the radius of the corona electrode and was in the range of 5 to $10 \mathrm{~cm}$ (Lin and Adamiak, 2008). Altogether, 28 wired electrodes were installed at $7 \mathrm{~cm}$ intervals.

\section{Ground electrode and material motion mechanism}

Since the electrodes' configuration of the system was the wire and plate type, the plate electrode distance from the corona electrodes should be constant. Moreover, a $2.5 \mathrm{~m}$-long conveyor was designed and constructed in order to move the frozen material continuously in the system.

The surface of the conveyor belt was considered as ground electrode, which moved along the ladder-shaped electrodes underneath the wire electrodes. Due to the presence of food on the conveyor belt, its material should be hygienic and in agreement with the standards for food industry machines (BS EN ISO, 2014). It should also be electrically conductive, flexible, and have sufficient tensile strength. Considering these criteria, a 304 stainless steel spring sheet, which was $0.1 \mathrm{~mm}$ thick, was used as conveyor belt.

The approximate duration for thawing the food materials was between 45 and 60 min (Guo et al., 2014). Considering the effective length of the conveyor belt $(240 \mathrm{~cm})$, the remaining duration with the material on the belt could be controlled by adjusting the belt velocity. The maximum velocity was considered to determine the required power. The velocity was calculated to be $3.5 \mathrm{~cm} \mathrm{~min}^{-}$ ${ }^{1}$ by considering the minimum thawing duration (45 min). A $375 \mathrm{~W}$ three phase AC electromotor and gearbox (Ratio of 1:100) with a speed controller were used to drive the belt.

\section{High voltage connections}

Regarding the high voltage connections, the driver roller was used to transfer the electric current to the metal belt. The driver roller was made of 304 stainless steel, which was conductive and in contact with the conveyor belt. In order to insulate the surface of the roller from its axis and prevent the transfer of the electric current to the bearings, a polyamide cylinder was inserted inside the tube. The driven roller was made of polyamide, which is an electric insulator. To transfer the electric current to the conveyor belt, as shown in Figure 1, a fixed carbon electrode was mounted on the device chassis which was sliding in contact with the driver roller. To supply the voltage, a DC power supply (Model HV50N OC, FNM co., ltd, Tehran, Iran) was utilized with voltage adjusting capability in the range of 0 to $70 \pm 1 \mathrm{kV}$ and a maximum current of $2000 \pm 1 \mu \mathrm{A}$. By connecting the poles of the power supply to the carbon electrode and the rails, the electric field between the two poles was created.

By applying the voltage and measuring the current in the system, the specific energy consumption (SEC) of the process was calculated by Eq. (3):

$$
S E C=\frac{V . I . t}{m}
$$

where $V$ was the applied voltage, $I$ was the electric current, $t$ was the thawing duration, and $m$ was the mass of the frozen material.

\section{Device chamber}

In order to prevent the air flow from entering the corona wind zone, control temperature and protect the user from the risk of electric shock, the whole conveyor and its components were placed in an insulated chamber. The chamber was made of a pressed plastic sheet in the form of a tunnel measuring $50 \times 50 \times 270 \mathrm{~cm}$ (Figure 1). In order for the materials to go in and out of the tunnel, its two ends were open and covered with two thick pads. On the top of the tunnel, two windows were installed longitudinally to facilitate user access to the conveyor. The glass was mounted on the lateral side 
and on the top of the tunnel to see the material flowing inside the system. In order to control ambient temperature, the entire setup was placed in a room, which was kept at a constant temperature by a temperature control system. As illustrated in Figure 2, the room was made with nylon and iron rods. The inlet and outlet of the airflow into and out of the room were placed on its floor in order to prevent the air flow from entering the device chamber and interfering with the corona wind. The temperature control sensor was mounted on the interior wall of the room at the same level of the device conveyor. Room temperature was set at $25 \pm 1^{\circ} \mathrm{C}$ during the experiments.

\section{Experiments}

To conduct the experiments, a tylose gel MH4000 sample was used with a moisture content of $77 \%$ w.b., since its thermo-physical properties are similar to red meat (Icier and Ilicali, 2005). The tylose gel sample was frozen within the range of -8 to $-10^{\circ} \mathrm{C}$ with a dimension of $1.5 \times 4 \times 6 \mathrm{~cm}$ and mass of $21.6 \pm 0.2 \mathrm{~g}$. The criterion of the thawing process was to increase the temperature of the sample centre to $0^{\circ} \mathrm{C}$. A 4 channel fibre optic thermometer (Model FOB100, Canada) equipped with multi-purpose fibre optic temperature sensors with the accuracy of $\pm 0.8^{\circ} \mathrm{C}$ was used to measure the temperature of the frozen sample centre and record it at $2 \mathrm{~s}$ intervals during the thawing process. For this purpose, the frozen gel was drilled with a $2 \mathrm{~mm}$ drill and the sample was placed on the conveyor belt after inserting the optical fibre. The experiments were conducted with the field and without the field as control. Thawing experiments were performed independently three times in this study and the average was calculated. In each HVEF treatment, the sample was placed in the same position on the grounded electrode for the sake of consistency.

Both the centre and surface temperatures of sample increased rapidly with an increase in the applied voltages and showed some similar characteristics (He et al., 2014). Therefore, we only measured the centre temperature of the tylose gel to investigate the effect of process parameters and electrode configuration. As to the thawing duration, the speed of the conveyor was adjusted to transfer the frozen material along the conveyor. The thawing duration was determined at voltages of 15,20 , and $25 \mathrm{kV}$, and EGs of 6,8 and $10 \mathrm{~cm}$. The distance between the adjacent wire electrodes was also $7 \mathrm{~cm}$.

\section{Statistical analysis}

The experiments were replicated three times based on a completely random design. The treatments were high voltages and EGs. The averages were compared according to the LSD test at a $5 \%$ probability level.

\section{Results and discussion}

To evaluate the designed and constructed system under the conditions mentioned above, the threshold value for the current in the power supply was set at $1000 \mu \mathrm{A}$. It should be noted that by applying the voltage to the electrodes, the current value should not exceed the threshold value. Moreover, by applying the higher voltages for an EG of $6 \mathrm{~cm}$ or adjusting the current threshold value to values greater than $1000 \mu \mathrm{A}$, an electrical discharge may occur between the two poles which prevents creating the corona wind.

Figure 3 shows variations in the temperature of the tylose gel sample over time in the presence of the electric field and without it (control). Differences were observed between thawing duration in the control and HVEF states. The HVEF conditions for thawing were the voltages of 15,20 , and $25 \mathrm{kV}$, the distance of $7 \mathrm{~cm}$ between adjacent wire electrodes, and an EG of 6,8 , and $10 \mathrm{~cm}$. As depicted, when using the control setup, the thawing duration was mostly devoted to increase the temperature, which considerably decreased, when thawing the sample in the presence of the high voltage. The thawing rate changed at three stages: before $6^{\circ} \mathrm{C}$, from -6 to $-3^{\circ} \mathrm{C}$, and from -3 to $0^{\circ} \mathrm{C}$ at all EGs and applied voltages. Obviously, the freezing point of the water solution in the food material is around -3 to $-2.8^{\circ} \mathrm{C}$ is. The second stage which is near the temperature of $-3^{\circ} \mathrm{C}$ is time consuming as it is close to the thawing point of the tylose sample, because of the latent heat requirement.

The values of electric current, electric field, thawing duration and specific energy consumption at different applied voltages and distances between the two poles are given in Table 1. As presented, the electric current increased with the applied voltage at a constant $\mathrm{EG}$, and decreased as the EG increased, when the voltage was fixed. There was a remarkable difference between control sample and the thawing process with the HVEF. In addition, the thawing duration significantly influenced by the high voltage and EG

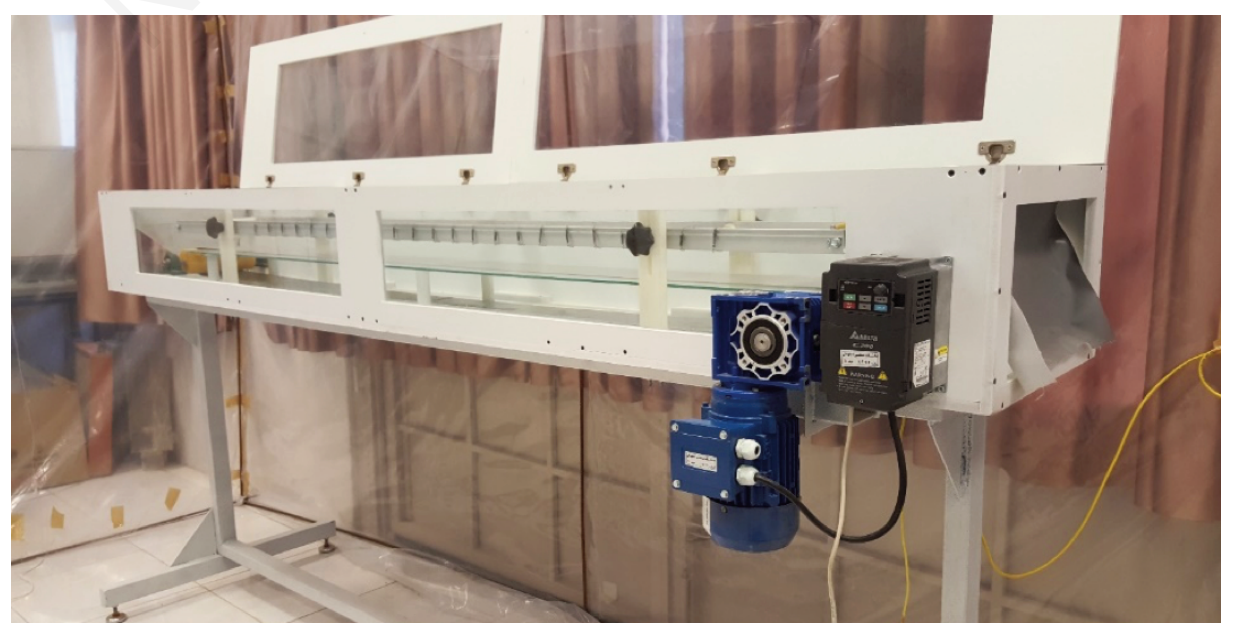

Figure 2. Continuous high voltage electrostatic field thawing system inside the nylon room. 
$(\mathrm{P}<0.0001)$. Among all the HVEF treatments, the longest thawing duration occurred with a voltage of $15 \mathrm{kV}$ and an $\mathrm{EG}$ of $10 \mathrm{~cm}$. The shortest thawing duration was with a voltage of $25 \mathrm{kV}$ and an EG of $6 \mathrm{~cm}$. These values decreased by about $33.5 \%$, and $76.3 \%$ compared to the corresponding values for the control, respectively.

The thawing duration decreased by decreasing the EG and increasing the applied voltage significantly (Table 1). Xiangli et al. (2013) studied the thawing of pork tenderloin meat under HVEF and reported that as voltage increased, the thawing duration decreased. This result was also found in another study (Xie and Hua, 2001). Yaxiang et al. (2009), and Yaxiang et al. (2011) showed that when the distance between the two adjacent electrodes was fixed, the ice thawing rate changed in an inversely proportional manner compared to the EG.

Similar to the thawing duration, the effect of high voltage and EG on the SEC was significant $(\mathrm{P}<0.0001)$. The SEC increased by decreasing the EG and increasing the applied voltage significantly (Table 1). This finding was also reported in previous researches (Yaxiang et al., 2009; Yaxiang et al., 2011).

According to Eq. (3) and as expected, the specific energy consumption is proportional to the thawing duration. However, the opposite trend for the thawing duration and SEC is due to the predominant effect of the reduction in the electric current, when the EG increases at a constant voltage. Hence, unlike the thawing

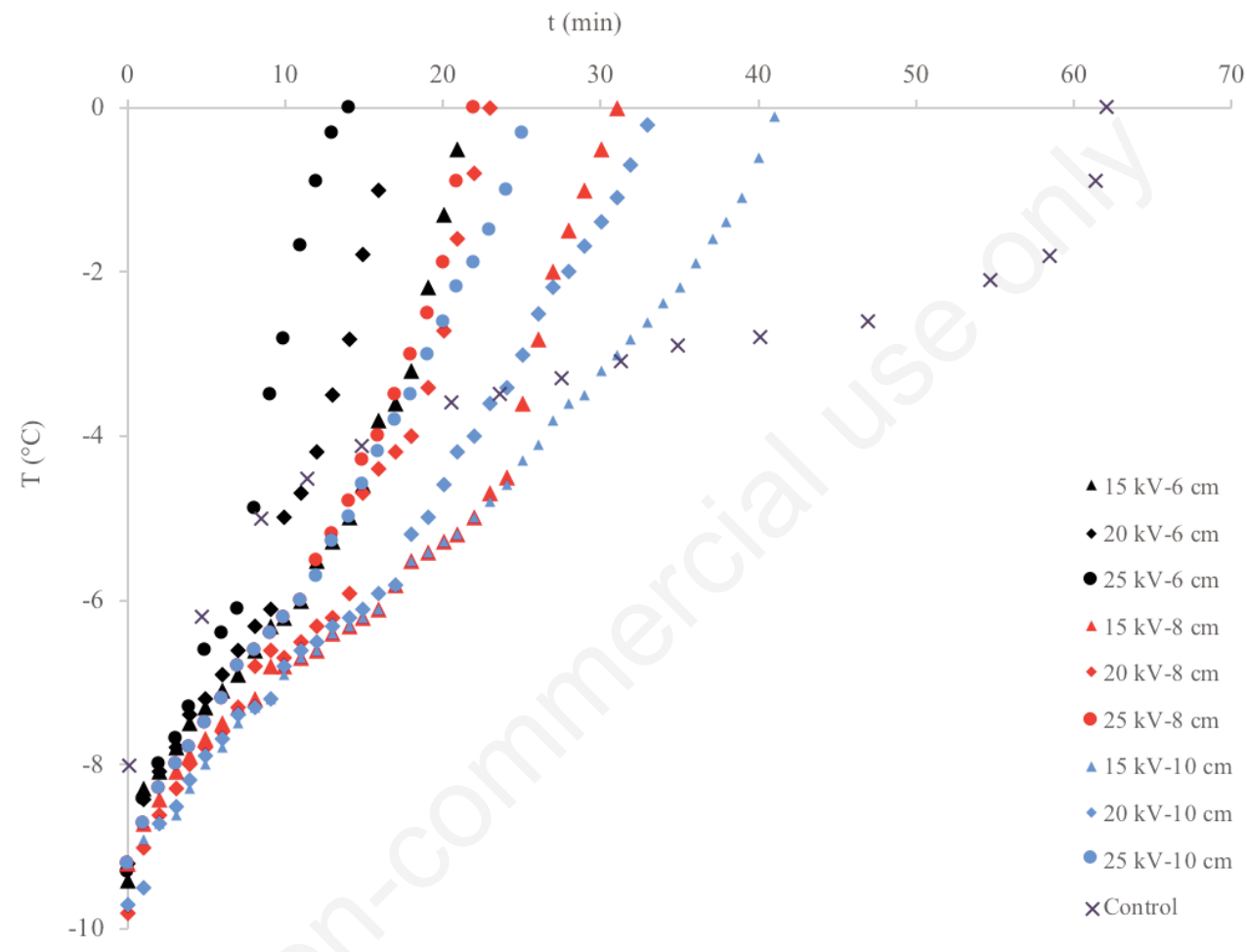

Figure 3. Variations in temperature of the tylose gel centre with thawing duration using the control setup and high voltage electrostatic field at different voltages $(15,20$, and $25 \mathrm{kV})$ and electrode gaps $(6,8$, and $10 \mathrm{~cm})$.

Table 1. Measured and calculated values of electric current, electric field, thawing duration and specific energy consumption for high voltage electrostatic field thawing of tylose gel at different voltages and electrode gaps.

\begin{tabular}{|c|c|c|c|c|c|}
\hline $\begin{array}{l}\text { Voltage } \\
(\mathrm{kV})\end{array}$ & $\begin{array}{l}\text { The electrode gap } \\
(\mathrm{cm})\end{array}$ & $\begin{array}{l}\text { Electric field } \\
\qquad\left(\mathrm{kV} \mathrm{m}^{-1}\right)\end{array}$ & $\begin{array}{l}\text { Electric current } \\
\qquad(\mu \mathrm{A})\end{array}$ & $\begin{array}{l}\text { Thawing duration } \\
\text { (min) }\end{array}$ & $\begin{array}{c}\text { SEC } \\
\left(\mathrm{kJ} \mathrm{kg}^{-1}\right)\end{array}$ \\
\hline 15 & $\begin{array}{c}6 \\
8 \\
10\end{array}$ & $\begin{array}{c}250 \\
187.5 \\
150\end{array}$ & $\begin{array}{c}24 \\
13 \\
6\end{array}$ & $\begin{array}{l}22.13 \pm 0.12^{\mathrm{g}} \\
31.30 \pm 0.32^{\mathrm{d}} \\
41.23 \pm 0.15^{\mathrm{b}}\end{array}$ & $\begin{array}{c}22.13 \pm 0.12^{\mathrm{f}} \\
16.97 \pm 0.18^{\mathrm{fg}} \\
10.33 \pm 0.03^{\mathrm{g}}\end{array}$ \\
\hline 20 & $\begin{array}{c}6 \\
8 \\
10 \\
\end{array}$ & $\begin{array}{c}333.3 \\
250 \\
200 \\
\end{array}$ & $\begin{array}{c}166 \\
30 \\
12 \\
\end{array}$ & $\begin{array}{l}17.50 \pm 0.26^{\mathrm{h}} \\
23.43 \pm 0.20^{\mathrm{f}} \\
33.40 \pm 0.17^{\mathrm{c}}\end{array}$ & $\begin{array}{l}161.40 \pm 2.44^{\mathrm{c}} \\
39.07 \pm 0.35^{\mathrm{e}} \\
22.30 \pm 0.12^{\mathrm{f}}\end{array}$ \\
\hline 25 & $\begin{array}{c}6 \\
8 \\
10\end{array}$ & $\begin{array}{c}416.6 \\
312.5 \\
250\end{array}$ & $\begin{array}{c}357 \\
178 \\
85\end{array}$ & $\begin{array}{c}14.67 \pm 0.24^{\mathrm{i}} \\
22.60 \pm 0.31 \mathrm{f}^{\mathrm{f}} \\
25.50 \pm 0.26^{\mathrm{e}}\end{array}$ & $\begin{array}{l}363.60 \pm 5.98^{\mathrm{a}} \\
279.33 \pm 3.79^{\mathrm{b}} \\
150.53 \pm 1.56^{\mathrm{d}}\end{array}$ \\
\hline Control & - & 0 & 0 & $62.08 \pm 0.57^{a}$ & - \\
\hline
\end{tabular}

The values for thawing duration and SEC present a mean \pm one standard error. ${ }^{\text {a-In }}$ each column, the means followed by the same common lowercase letter do not differ statistically based on the LSD test with a $5 \%$ probability level. 
duration, the highest value of the SEC was achieved at a voltage of $25 \mathrm{kV}$ and an EG of $6 \mathrm{~cm}$, while the lowest value occurred at a voltage of $15 \mathrm{kV}$ and an $\mathrm{EG}$ of $10 \mathrm{~cm}$.

\section{Conclusions}

In this study, a high voltage electrostatic field continuous thawing system in wire-plate electrode setups was designed and constructed for thawing foodstuffs according to the standards of food industry machines. The device was evaluated using frozen tylose MH4000, which is a meat analogue in order to investigate the feasibility of foodstuff thawing with this device. For this assessment high voltages of 10,15 , and $25 \mathrm{kV}$ and EGs of 6,8 , and $10 \mathrm{~cm}$ were considered. Based on our results, the HVEF continuous system proved to be a feasible rapid method for thawing of food materials such as meat. As the system was developed on a laboratory scale, further studies are needed the future to assess its energy consumption in comparison with the other thawing systems and its economic feasibility for industrial scale applications in the food sector.

\section{References}

Akishev Y.S., Dem'yanov A.V., Karal'nik V.B., Monich A.E., Trushkin N.I. 2003. Comparison of the AC barrier corona with DC positive and negative coronas and barrier discharge. Plasma Phys. Rep. 29:82-91.

BS EN 453:2014. Food processing machinery. Dough mixers. Safety and hygiene requirements. Available from: https://doi.org/10.3403/30251966

Cai L., Cao M., Regenstein J., Cao A. 2019. Recent advances in food thawing technologies. J. Compr. Rev. Food Sci. Food Saf. 18:953-70.

Deng S., Gao Z., Xu J., Wang G., Bai Y., Ding C. 2017. The thawing characteristic of frozen tofu under high-voltage alternating electric field. J. Food Qual. 2017:1-6.

Ding S., Ni J., Song Z., Gao Z., Deng S., Xu J., Wang G., Bai Y. 2018. High voltage electric field-assisted thawing of frozen tofu: effect of process parameters and electrode configuration. J. Food Qual. 2018:1-8.

Eastridge J.S., Bowker B.C. 2011. Effect of rapid thawing on the meat quality attributes of USDA select beef strip loin steaks. J. Food Sci. 76:156-62.

Guo B.Y., Guo J., Yu A.B. 2014. Simulation of the electric field in wire-plate type electrostatic precipitators. J. Electrostat. 72, 301-10.
He X., Liu R., Nirasawa S., Zheng D., Liu H. 2013. Effect of high voltage electrostatic field treatment on thawing characteristics and post-thawing quality of frozen pork tenderloin meat. J. Food Eng. 115:245-50.

He X., Liu R., Tatsumi E., Nirasawa S., Liu H. 2014. Factors affecting the thawing characteristics and energy consumption of frozen pork tenderloin meat using high-voltage electrostatic field. Innov. Food Sci. Emerg. Technol. 22:110-5.

Hsieh C.W., Lai C.H., Ho W.J., Huang S.C., Ko W.C. 2010. Effect of thawing and cold storage on frozen chicken thigh meat quality by high-voltage electrostatic field. J. Food Sci. 75:193-7.

Icier F., Ilicali C. 2005. The use of tylose as a food analog in ohmic heating studies. J. Food Eng. 69:67-77.

Jia G., Nirasawa S., Ji X., Luo Y., Liu H. 2017. Physicochemical changes in myofibrillar proteins extracted from pork tenderloin thawed by a high-voltage electrostatic field. Food Chem. 240:910-6.

Lin Z., Adamiak K. 2008. Numerical simulation of the electrohydrodynamic flow in a single wire-plate electrostatic precipitator. IEEE. Trans. Ind. Appl. 44:683-91.

Ohtsuki T. 1991. Process for thawing foodstuffs. US Patent 5034236 .

Ould Ahmedou S.A., Havet M. 2009. Effect of process parameters on the EHD airflow. J. Electrostat. 67:222-7.

Shivashankara K.S., Isobe S., Al-Haq M.I., Takenaka M., Shiina T. 2004. Fruit antioxidant activity, ascorbic acid, total phenol, quercetin, and carotene of Irwin mango fruits stored at low temperature after high electric field pretreatment. J. Agric. Food Chem. 52:1281-6.

Taher B.J., Farid M.M. 2001. Cyclic microwave thawing of frozen meat: experimental and theoretical investigation. Chem. Eng. Process. 40:379-89.

Xiangli H., Rui L., Nirasawa S., Zheng D., Liu H. 2013. Effect of high voltage electrostatic field treatment on thawing characteristics and post-thawing quality of frozen pork tenderloin meat. J. Food Eng. 115:245-50.

Xie J., Hua Z.Z. 2001. The experimental research on the thawing process of quickly frozen potato in the high static-electric voltage fields. J. Refr. 2:1-5 [In Chinese].

Yar R., Faye Bedane T., Erdogduc F., Koray Palazoglu T., Farag K.W., Marra F. 2015. Radio-frequency thawing of food products - A computational study. J. Food Eng. 146:163-71.

Yaxiang B., Luan Z.Q., Li X.J., Xu J.P. 2009. Study on the thawing mechanism of high voltage electrostatic field. Trans. CSAE. 26:347-50.

Yaxiang B., Sun Y., Li Z., Kang D. 2011. Study the optimum parameters of high voltage electrostatic field thawing. Procedia Eng. 16:679-84. 